\title{
Editorial
}

\section{Variational Methods and Critical Point Theory 2013}

\author{
M. Victoria Otero-Espinar, ${ }^{1}$ Juan J. Nieto, ${ }^{1,2}$ Donal O'Regan, ${ }^{3}$ and Kanishka Perera ${ }^{4}$ \\ ${ }^{1}$ Departamento de Análise Matemática, Facultade de Matemáticas, Universidade de Santiago de Compostela, Galicia, \\ 15782 Santiago de Compostela, Spain \\ ${ }^{2}$ Department of Mathematics, Faculty of Science, King Abdulaziz University, P.O. Box 80203, Jeddah 21589, Saudi Arabia \\ ${ }^{3}$ School of Mathematics, Statistics and Applied Mathematics, National University of Ireland, Galway, Ireland \\ ${ }^{4}$ Department of Mathematical Sciences, Florida Institute of Technology, Melbourne, FL 32901, USA
}

Correspondence should be addressed to M. Victoria Otero-Espinar; mvictoria.otero@usc.es

Received 1 December 2013; Accepted 1 December 2013

Copyright ( 2013 M. Victoria Otero-Espinar et al. This is an open access article distributed under the Creative Commons Attribution License, which permits unrestricted use, distribution, and reproduction in any medium, provided the original work is properly cited.

Critical Point Theory and Variational Methods are crucial in the study of many mathematical models of real-world problems. Many applied problems can be understood and solved in terms of the minimization of a functional, usually related to the energy, in an appropriate functional space. Minimization and variational problems are at the interface between nonlinear analysis, calculus of variations, differential equations, and mathematical physics and play a fundamental role in the application of mathematics to different scientific areas.

This new special issue places its emphasis on the study and application of variational methods and critical point theory. The editors aim was that this volume first serves as an updated reference in the topic and second collects several papers by specialists in this area and from many different researchers.

This special issue focuses on new and recent developments on the theory, methods, and applications of boundary value problems. As requested in the call for papers, it contains papers on

(i) variational methods;

(ii) critical point theory;

(iii) Morse theory;

(iv) Lusternik-Schnirelmann theorem;

(v) variational inequalities;

(vi) ordinary differential equations;

(vii) partial differential equations; (viii) difference equations;

(ix) impulsive and shock dynamical systems;

(x) equations on Riemann manifolds;

(xi) applications to physics, economics, optimal control, engineering, industrial mathematics, biology, and medicine;

(xii) computational and numerical methods.

In this special issue, there are papers which consider

(i) fuzzy variationl inequalities;

(ii) sensitivity of fractional integrodifferential problems;

(iii) impulsive differential equations with nonlinear derivative dependence using variational methods;

(iv) minimax results with respect to different altitudes in the situation of linking;

(v) homoclinic solutions for second-order-Laplacian differential system or for second-order impulsive Hamiltonian systems;

(vi) positive periodic solutions of generalized-species competition systems with multiple delays and impulses;

(vii) existence of prescribed-norm solutions for a class of Schrödinger-Poisson equation and standing wave solutions for discrete nonlinear Schrödinger equations. 
Evidently, it is not possible to adequately represent in this special issue all directions of current research in variational methods and critical point theory, but we believe that it reflects many important recent trends in research, indicates current challenging problems, and outlines new ideas and open problems for future studies.

\section{Acknowledgments}

The editors of this special issue would like to express their gratitude to the authors who have submitted manuscripts for consideration. They also thank the many individuals who served as referees of the submitted manuscripts. All the participants have made it possible to have a very stimulating interchange of ideas. They hope that these topics will stimulate future research.

M. Victoria Otero-Espinar Juan J. Nieto Donal O'Regan Kanishka Perera 


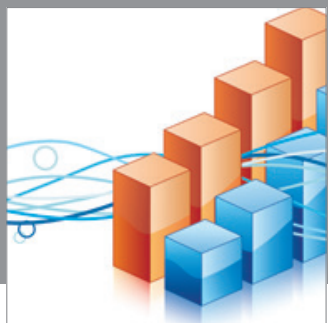

Advances in

Operations Research

mansans

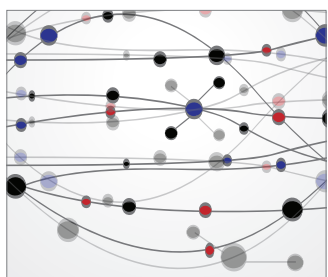

The Scientific World Journal
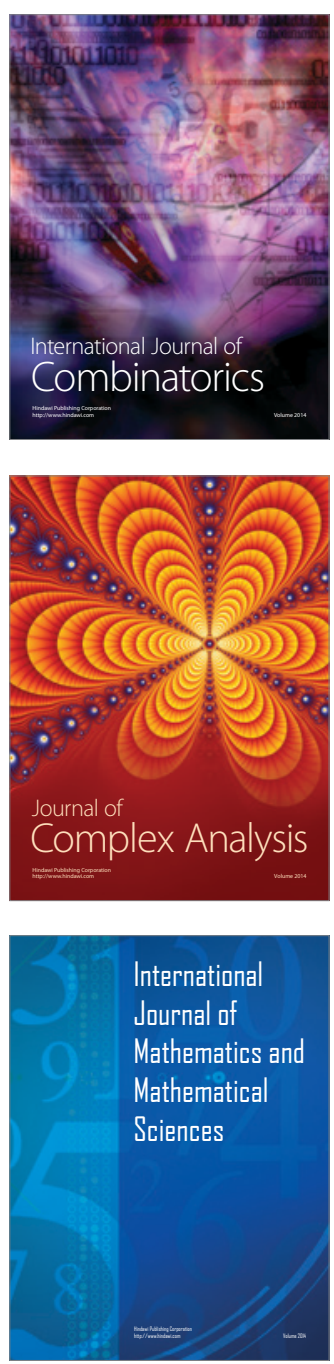
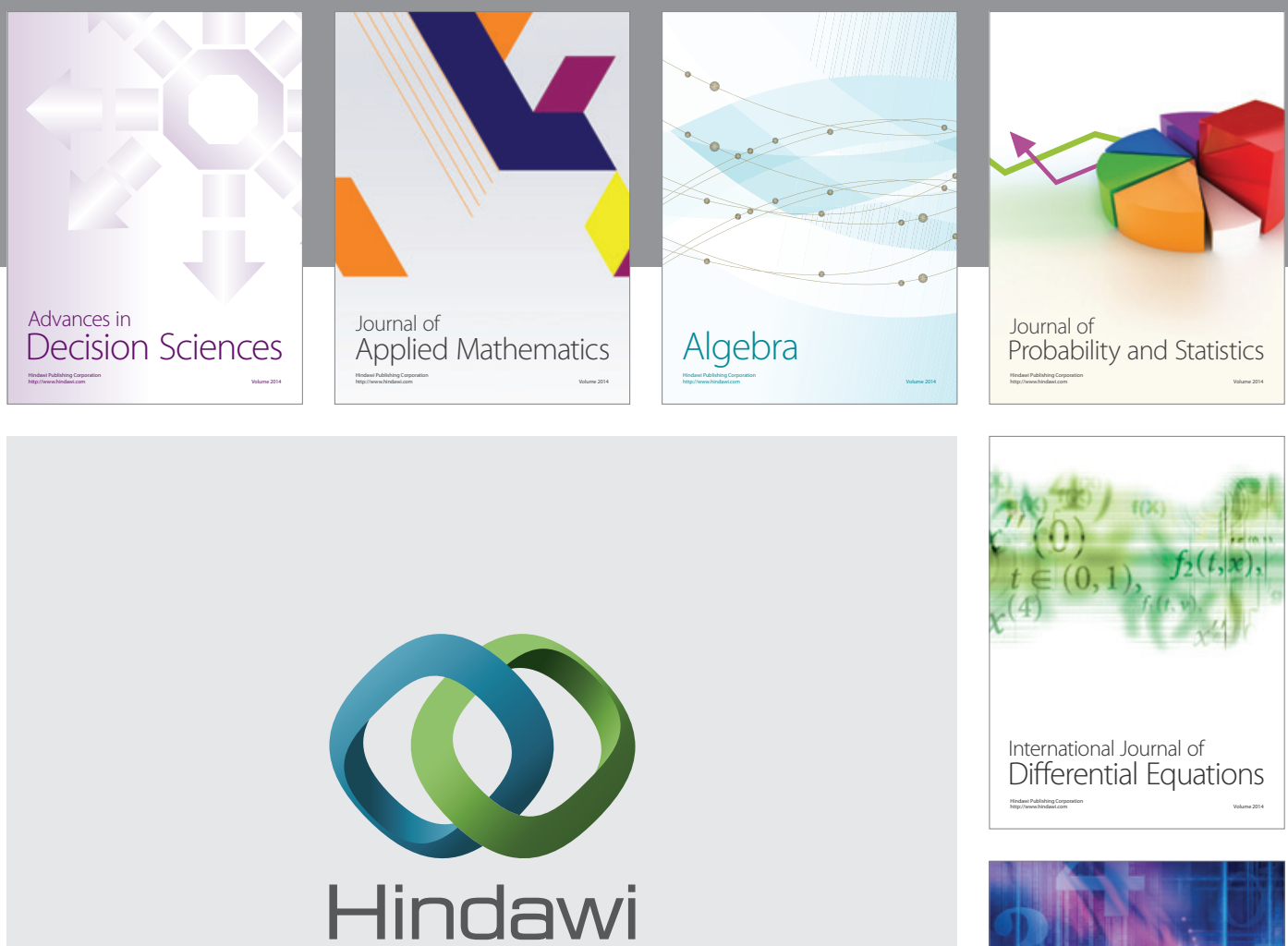

Submit your manuscripts at http://www.hindawi.com
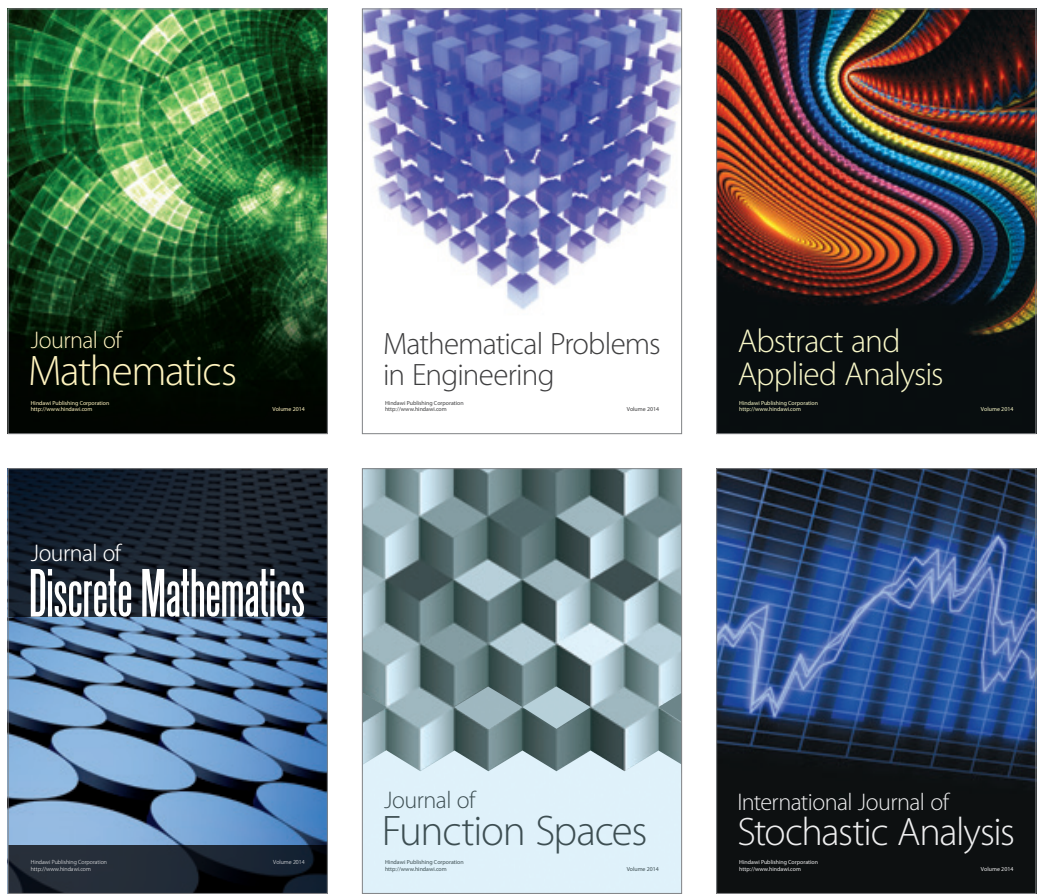

Journal of

Function Spaces

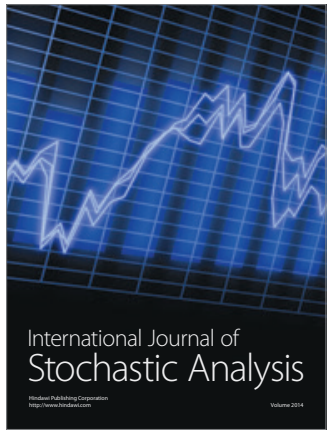

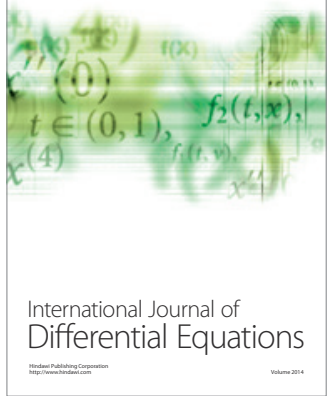
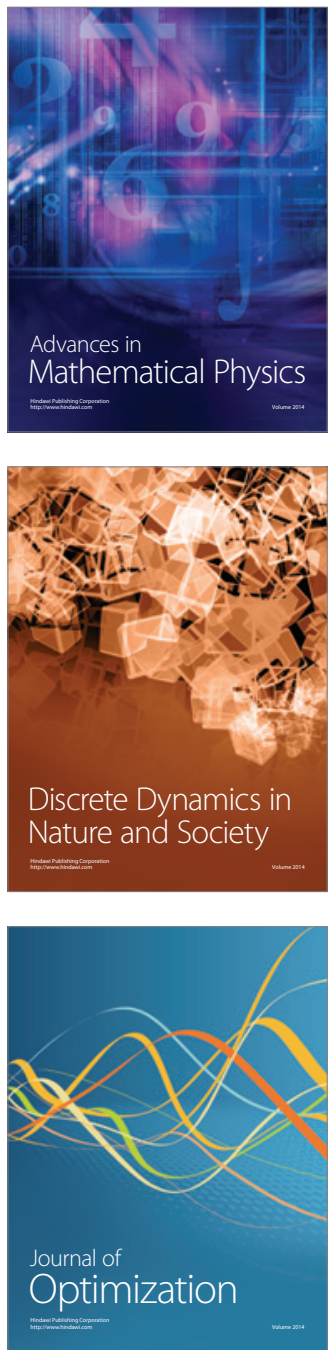